\title{
Alcohol septal ablation in hypertrophic cardiomyopathy utilizing a longitudinal 17-year study (mean 10.8). Observation follow-ups taken at a single medical centre
}

\author{
Andrzej Wojtarowicz, Zdzisława Kornacewicz-Jach \\ Department of Cardiology, Pomeranian Medical University, Szczecin, Poland
}

\begin{abstract}
Background: Alcohol septal ablation (ASA) is a method of treatment in obstructive hypertrophic cardiomyopathy (HOCM), but there is little data on the long-term results of ASA and the natural course after treatment. The aim of the study was to evaluate the results of ASA in HOCM in multiannual observation, and its impact on patient survival, exercise capacity, electrical complications, and changes in the anatomy and function of the heart.

Methods: The study evaluated 47 patients with HOCM with a high left ventricular outflow tract (LVOT gradient) treated between 1997 and 2014 with ASA. Annual examinations evaluated the clinical condition, at rest and with exercise electrocardiogram, Holter monitoring, echocardiography, the evolution of HOCM towards the dilated form, and the frequency of pacemaker implantation.

Results: The analysis included data from 34 patients under observation for 3 to 17 (mean 10.8) years. Their age at procedure was 21-65, a mean of 47 years. All patients had permanently reduced LVOT gradient with a mean of $77.36 \pm 35.46$ to $11.40 \pm 10.85$ and showed improvement in the performance I to II New York Heart Association. Two out of five deaths had possible cardiac etiology. Fifteen patients received a pacemaker or cardioverter implants. In 4 subjects the long-term observation revealed new wall contractility abnormalities, interpreted as a shift of HOCM to the dilated form.

Conclusions: Alcohol septal ablation permanently eliminated the gradient in LVOT and improved the performance of patients, however it did not prevent a shift of HOCM to the dilated form. Pacemaker implantations are relatively frequent. (Cardiol J 2017; 24, 2: 125-130)
\end{abstract}

Key words: alcohol septal ablation, arrhythmias and conduction abnormalities, heart failure, hypertrophic obstructive cardiomyopathy

\section{Introduction}

Hypertrophic cardiomyopathy $(\mathrm{HCM})$ is one of the most common heart diseases with a genetic background. It occurs in approximately $0.2 \%$ of the population; approximately $50 \%$ can be attributed to family burden $[1,2]$. The prognosis is relatively good for most patients; in mild forms, the risk of severe cardiac complications (sudden death, dan- gerous arrhythmias) is slightly higher than in the healthy population. The clinical symptoms most often occur as a result of left ventricular (LV) diastolic dysfunction or heart rhythm disorders. Patients with LV outflow track (LVOT) gradient (hypertrophic obstructive cardiomyopathy [HOCM]) in the course of HCM form a particular group. This group of patients often present symptoms of heart failure; they have a greatly increased tendency for

Address for correspondence: Andrzej Wojtarowicz, MD, PhD, Professor of the Pomeranian Medical University, Department of Cardiology, Pomeranian Medical University, Al. Powstańców Wielkopolskich 72, 70-111 Szczecin, Poland, tel: +48 91466 1377, fax: +48 91466 1379, e-mail: wojtaro@pum.edu.pl

Received: 24.02.2016 Accepted: 21.07.2016 
serious arrhythmia, and may have characteristics of low cardiac output, especially during exercise. This form of HCM has a significantly worse prognosis regarding life expectancy, and clearly impairs patient's quality of life. While non-obstructive forms of HCM medications (beta-blockers, verapamil) may have a positive impact on the clinical condition of patients, in the obstructive forms, the possibilities of pharmacotherapy are limited. It should be noted, that over a period of many years, classic HCM can transform into the dilated form leading to clinical heart failure, regardless of the primary form of the disease $[3,4]$. HCM without the obstruction of the LVOT does not require interventional treatment [5], while a significant narrowing of the outflow tract is an indication for intervention cardiac surgery (excision of the segment of the interventricular septum responsible for the gradient and possible mitral valve repair), or closing - usually by alcohol ablation — the artery supplying the segment of the interventricular septum (IVS) responsible for the gradient, or, when there are contraindications to the above mentioned procedures, 2-chamber pacemaker implantation $[6,7]$.

The first myectomy operations in HOCM were performed in the early 1960s [8]. This procedure requires open-heart surgery, and presents a risk of IVS perforation. This procedure does, however, offer the possibility of simultaneous repair of the mitral valve dysfunction leading to an immediate reduction of the gradient in LVOT. Inducing controlled myocardial infarction in the segment of the IVS responsible for the gradient in LVOT by the administration of $98 \%$ ethanol (alcohol septal ablation [ASA]) to the appropriate septal branch of the anterior descending artery is an alternative to this procedure, and has comparable effectiveness. This causes necrosis of the IVS areas supplied by this artery, reduction in the thickness and/or the formation of scar tissue in this area, and liquidation or significant reduction in the gradient in LVOT. The most common complication is intraventricular conduction disturbances requiring pacemaker implantation. The first ASA procedures in humans were carried out in 1995 [9]. In July 1997 at the Pomeranian Medical University, Szczecin, Poland the first ASA series on 7 patients was performed [10].

\section{Methods}

Alcohol septal ablation was applied on patients who met the criteria, i.e. obstruction LVOT with rest or provoked gradient $>60 \mathrm{~mm} \mathrm{Hg}$ and the clinical symptoms of at least New York Heart Association (NYHA) class II despite optimal pharmacotherapy. An additional eligibility criterion was septal thickness in part responsible for a gradient of at least $17 \mathrm{~mm}$ and the lack of hemodynamically significant mitral insufficiency. The ultimate eligibility criterion for the treatment was the anatomy of coronary arteries responsible for the gradient in LVOT allowing for the procedure. The management protocol included an initial clinical assessment (NYHA class, fainting, electrocardiogram [ECG], Holter monitoring, ECG exercise test) and echocardiography (gradient in LVOT at rest and after provocation, anatomy, systolic and diastolic function of the LV, mitral valve function) and coronary angiography. The control tests included echocardiography, ECG and biochemical tests immediately after ASA, and during the first week after clinical trial monitoring, echocardiography, Holter monitoring, and ECG exercise tests were conducted after 3, 6, and 12 months, and then every year thereafter, more often if necessary. The first 20 patients were subjected to an evaluation of the gradient in LVOT by catheterization 6 months after ASA; the remaining patients were examined using echocardiography. The study analyzed the ECG changes, and the occurrence of arrhythmias and/or conduction disorders, especially in terms of the need for electrotherapy, including the implantation of a pacemaker or cardioverter-defibrillator (ICD), exercise capacity, and changes in echocardiography. The analysis also included the symptoms of other systems and organs that occurred during the observation. From 1997 to the present, 55 people were enrolled for the procedure; ASA was performed in $47(85.5 \%)$ patients. Seven patients were excluded from the procedure due to coronary artery anatomy; one did not have ablation due to advanced atherosclerotic lesions in the coronary arteries. The maximum follow-up time was 17 years; the mean time was 10.7 years. This detailed analysis has included the results from patients over a minimum 3 years of observation and contains data from 34 patients. Data on the length of the observation is shown in Table. 1. Treatment was deemed successful if the gradient in LVOT was abolished or if it was reduced at rest and after provocation to below $30 \mathrm{~mm} \mathrm{Hg}$.

The study was approved by the local bioethical committee and all patients gave their informed consent. 
Table 1. The duration of follow-up in individual patients.

\begin{tabular}{lc}
\hline $\begin{array}{l}\text { Duration of } \\
\text { follow-up [years] }\end{array}$ & $\begin{array}{c}\text { Number of } \\
\text { patients }\end{array}$ \\
\hline 17 & 3 \\
16 & 2 \\
15 & 3 \\
14 & 3 \\
13 & 2 \\
12 & 3 \\
11 & 5 \\
10 & 2 \\
8 & 2 \\
7 & 1 \\
6 & 1 \\
5 & 5 \\
4 & 2 \\
3 & 1 \\
\hline
\end{tabular}

Table 2. The number of patients with New York Heart Association (NYHA) performance class before alcohol septal ablation, during the period of maximum improvement after procedure and the last follow-up.

\begin{tabular}{lccc}
\hline Class & $\begin{array}{c}\text { Initial } \\
\text { NYHA }\end{array}$ & $\begin{array}{c}\text { Optimal } \\
\text { NYHA }\end{array}$ & $\begin{array}{c}\text { Final } \\
\text { NYHA }\end{array}$ \\
\hline III/IV & 2 & & \\
III & 16 & & \\
II/III & 10 & 1 & 3 \\
II & 6 & 2 & 14 \\
I/II & & 4 & 4 \\
I & & 27 & 13 \\
\hline
\end{tabular}

\section{Results}

The values of NYHA at the beginning and during the follow-up are shown in Table 2. Before the procedure episodes of syncope occurred in $9(26.5 \%)$ patients. There were 5 deaths in the whole study group, including 2 of possible cardiac etiology (1 patient — sudden death 9 months after the procedure, and 1 patient with atrial fibrillation 12 months after the procedure - possibly stroke). Other causes included: in 1 case - a traffic accident, and in 2 cases - cancer. Contact was lost with 12 patients or they refused further follow-up examinations. The final analysis included the results from 34 patients with a mean age of 47 (21-65) years at the moment of ASA. At least 3 months after ablation all subjects achieved a significant improvement in their clinical condition, and almost all had NYHA class I.

The results of echocardiography are shown in Table 3. All patients demonstrated liquidation or significant reduction of the gradient in LVOT, though in 1 patient the final result of ASA (complete disappearance of the gradient in LVOT) occurred only 1 year after procedure and in another it was necessary to perform re-treatment and closure of another septal artery. In the whole study group (excluding the 2 cases mentioned above) the final gradient in LVOT established after 3-6 months and during further follow-up did not increase. All patients manifested increased end-systolic and 32 end-diastolic dimension of the LV. The thickness of the part located at the base of IVS was reduced in all patients; in $12(35.3 \%)$ patients a post-infarction scar appeared at the site of ablation. The thickness of the posterior wall of the LV in the whole study group was also significantly reduced. Basic parameters of the LV diastolic function did not change, aside from the extension of isovolumetric relaxation time.

New atrioventricular and intraventricular conduction disturbances were observed in most individuals. During the procedure and in the early period after ASA, 24 patients had right bundle branch block (RBBB), 5 left bundle branch block (LBBB), and 11 patients had various types of atrio-ventricular blocks - from I to III grade. In $12(35.3 \%)$ patients further follow-up revealed fixed RBBB and in 3 (8.8\%) patients LBBB. Four $(11.8 \%)$ patients required pacemaker implantation immediately after ablation, and during further follow-ups lasting from 1 to 9 years after ASA $11(32.4 \%)$ consecutive patients had indications for pacemaker implantation. Eleven of the entire group of 15 individuals with an implanted pacing system were classified for implantation of an ICD or a change of a pacemaker to an ICD on the basis of existing standards, mainly due to ventricular arrhythmia. Atrial fibrillation was reported in a total of 12 patients - in 1 it was present before ASA, in $11(32.4 \%)$ the disorder was observed 2-9 years after ASA.

In $12(35.3 \%)$ of the ablated patients from 4 to 10 years after procedure, the symptoms of heart failure increased to class NYHA II. Atrial fibrillation during follow-up was reported in 9 (26.5\%) patients, $2-12$ years after ASA (mean 8.3 years); in 1 person the disorder was observed from the beginning of the follow-up. In $3(8.8 \%)$ patients 
Table 3. Basic echocardiographic parameters in the study group.

\begin{tabular}{|c|c|c|c|c|c|}
\hline & \multicolumn{2}{|c|}{ Initial examination } & \multicolumn{2}{|c|}{ Last follow-up } & \multirow[t]{2}{*}{$\mathbf{P}$} \\
\hline & Mean \pm SD & Range & Mean \pm SD & Range & \\
\hline LVOT gradient [mm Hg] & $77.36 \pm 35.46$ & $30-205$ & $11.40 \pm 10.85$ & $1.3-30$ & $<0.0001$ \\
\hline LVSD [cm] & $2.23 \pm 0.39$ & $1.56-3.18$ & $3.71 \pm 0.85$ & $2.3-5.3$ & $<0.0001$ \\
\hline LVDD [cm] & $4.18 \pm 0.46$ & $3.18-4.93$ & $5.18 \pm 0.74$ & $3.7-6.6$ & $<0.0001$ \\
\hline EF [\%] & $77.94 \pm 6.47$ & $62-90$ & $58.76 \pm 10.22$ & $32-78$ & $<0.0001$ \\
\hline IVSd [cm] & $2.30 \pm 0.49$ & $1.59-3.89$ & $1.37 \pm 0.45$ & $0.6-2.5$ & $<0.0001$ \\
\hline $\mathrm{PWd}[\mathrm{cm}]$ & $1.40 \pm 0.29$ & $1.03-2.26$ & $1.15 \pm 0.22$ & $0.9-1.9$ & $<0.0001$ \\
\hline $\mathrm{LA}[\mathrm{cm}]$ & $4.26 \pm 0.53$ & $3.01-5.34$ & $4.66 \pm 0.76$ & $3.0-6.4$ & 0.015 \\
\hline$E[\mathrm{~m} / \mathrm{s}]$ & $0.88 \pm 0.24$ & $0.46-1.36$ & $0.79 \pm 0.23$ & $0.40-1.69$ & NS \\
\hline$A[\mathrm{~m} / \mathrm{s}]$ & $0.76 \pm 0.26$ & $0.24-1.38$ & $0.76 \pm 0.26$ & $0.36-1.61$ & NS \\
\hline E/A ratio & $1.27 \pm 0.49$ & $0.59-2.38$ & $1.13 \pm 0.43$ & $0.56-2.09$ & NS \\
\hline IVRT [ms] & $94.68 \pm 20.19$ & 44-132 & $109.68 \pm 30.65$ & $48-180$ & 0.022 \\
\hline DT [ms] & $232.94 \pm 54.02$ & $133-362$ & $245.62 \pm 79.54$ & $127-501$ & NS \\
\hline MI scale 1-4 & $1.71 \pm 0.67$ & $0-3$ & $1.81 \pm 0.50$ & $1-3$ & NS \\
\hline
\end{tabular}

A - mitral A wave; DT - deceleration time of mitral E wave; E - mitral E wave; EF — ejection fraction; IVRT — isovolumetric relaxation time; IVSd - intraventricular septum thickness in diastole; LA — left atrium; LVOT — left ventricular outflow tract; LVDD — left ventricular diastolic diameter; LVSD — left ventricular systolic diameter; MI — mitral insufficiency; PWd — posterior wall thickness in diastole; SD - standard deviation

echocardiography found LV contractility impairment in the area of the inferior wall 7-10 years after ASA, as well as a significant decrease in ejection fraction; in 1 patient there was hypokinesis of the para-apical segments of the LV. Coronary angiography showed no significant stenosis, and therefore an evolution towards the dilated form of HCM was reported. During a follow-up of the study group the following conditions were diagnosed: 5 cases of cancer, 3 cases of coronary artery disease diagnosed for the first time, 2 - hyperthyroidism and 1 - hypothyroidism, 9 - diabetes, and $1-$ borreliosis.

\section{Discussion}

Hypertrophic cardiomyopathy is the most common genetic heart disease. It occurs in approximately 1 per 500 people, and in about $50 \%$ of these family burden can be established $[1,2]$. While the non-obstructive form often does not cause a significant impairment in exercise capacity (sometimes it even does not impede practising sport), the obstruction of the outflow from the LV can lead to severe clinical symptoms [6]. Regardless of the form, HCM is a significant risk factor for sudden cardiac death (SCD), but LVOT obstruction significantly increases this risk [11]. Ventricular arrhythmias are the most common cause of SCD in $\mathrm{HCM}$, especially in the obstructive form $[12,13]$.
In follow-up heart failure may occur due to the progressive impairment of LV contractility $[4,13,14]$.

Liquidation or significant reduction in the gradient in LVOT not only improves the quality of life of patients with HOCM, but also significantly reduces the risk of SCD [15]. For around the last 20 years ASA (and similar methods of closing the coronary arteries which supply the IVS) has been an alternative to cardiac surgery in obstructive, symptomatic HCM. Although this is not an immediate countermeasure in the reduction or elimination in the gradient in LVOT [16], it is much less invasive and reduces the risks due to a number of complications. This method is recommended for experienced medical centres with an adequate diagnostic base and an experienced team who can perform this procedure [6]. Treatments around the world have been carried out for about 20 years now [9, 17], and at Pomeranian Medical University, Szczecin, Poland since 1997 [10]. According to literature data the efficacy of ASA is practically the same as surgical treatment of HOCM, the number of complications is similar with a slightly different profile, with indications also the same, so the choice of treatment depends on the experience of the medical centre $[18,19]$. The advantage of ASA includes being significantly less invasive; a disadvantage is the dependence on the anatomy of the coronary arteries, and in some cases an inability to improve mitral valve function. The smaller 
dynamics of the gradient reduction in LVOT is a secondary issue in the context of the natural history of the disease. There are few works assessing ASA efficacy in the long-term perspective through the course of the disease in treated patients and other concomitant diseases. Observation of a group of patients who undergo this treatment for a period more than 10 years is rare [20-24].

All patients in the present study achieved a lasting reduction of the gradient in LVOT below $30 \mathrm{~mm} \mathrm{Hg}$ within at least 3 years of observation, only 1 person required a repeated treatment after 1 year. Clinical improvement by at least I NHYA class was achieved in all study patients, and the effectiveness of the treatment assessed in terms of reducing the gradient and a significant improvement in clinical status was practically $100 \%$. Long-term observations of patients with HCM showed that some of these, regardless of the form of the disease, with time manifested impaired LV contractility, its widening, and evolution into a form resembling dilated cardiomyopathy. In the study group, a similar trend was observed in 4 patients who a few years after ablation developed a permanent decrease in ejection fraction with the areas of impaired contractility in the absence of coronary lesions. In these patients the efficacy of ablation was complete, and there was no residual gradient in LVOT, so the transition into the dilated form of $\mathrm{HCM}$ was diagnosed and observed in the natural history of this disease.

Indications for electrotherapy is the most important problem. In HCM, regardless of the form, already significant LV hypertrophy is an indication for ICD implantation [7, 24]. ASA (slightly more often than surgery) which leads to atrioventricular and intraventricular disorders (mainly RBBB), and in the long term can lead to the need for pacemaker implantation. In the study population indications for the implantation of a pacemaker or ICD were present in 15 people, including 4 in the peri-procedure period, and the remaining a period from 1 to 12 years after ASA. It is of note that eventually a device was implanted in as many as 11 patients, especially those with electrophysiological indications requiring ICD.

\section{Limitations on the study}

The patient study group was relatively small, and the observation period varied. Moreover, there was no possibility for a comparison with a similar group of patients with HOCM who underwent surgical treatment, and those with HOCM and a significant gradient in LVOT not undergoing interventional treatment. In addition, due to the long period of observation and changing equipment capabilities, it was not possible to compare more advanced echocardiographic parameters based on the tissue Doppler. Also, different protocols for exercise ECG tests were used. During follow-up of the study group, indications for electrotherapy changed, as well as electrophysiological treatment options. The regularity of control examinations was better in patients with an implanted pacing system, who were more aware of the need for systematic and periodic checks.

\section{Conclusions}

1. Alcohol septal ablation is a highly effective procedure for improving hemodynamics and the clinical status in HOCM for at least several years after the procedure. Over a longer period the clinical picture is significantly affected by diseases of other organs and random events.

2. Interventional treatment does not prevent the evolution of HCM into the dilated form.

3. Atrioventricular block requiring pacemaker implantation is the most common early complication in the study group. Despite the significant improvement in hemodynamics and in most cases beneficial LV remodeling, after an extended period a large group of patients had indications for the implantation of a pacemaker or ICD.

\section{Conflict of interest: None declared}

\section{References}

1. Maron BJ, Gardin JM, Flack JM, et al. Prevalence of hypertrophic cardiomyopathy in a general population of young adults. Echocardiographic analysis of 4111 subjects in the CARDIA Study. Coronary Artery Risk Development in (Young) Adults. Circulation. 1995; 92(4): 785-789, indexed in Pubmed:7641357.

2. Richard P, Charron P, Carrier L, et al. EUROGENE Heart Failure Project. Hypertrophic cardiomyopathy: distribution of disease genes, spectrum of mutations, and implications for a molecular diagnosis strategy. Circulation. 2003; 107(17): 2227-2232, doi: 10.1161/01.CIR.0000066323.15244.54, indexed in Pubmed: 12707239 .

3. Melacini P, Basso C, Angelini A, et al. Clinicopathological profiles of progressive heart failure in hypertrophic cardiomyopathy. Eur Heart J. 2010; 31(17): 2111-2123, doi: 10.1093/eurheartj/ehq136, indexed in Pubmed: 20513729.

4. Harris KM, Spirito P, Maron MS, et al. Prevalence, clinical profile, and significance of left ventricular remodeling in the endstage phase of hypertrophic cardiomyopathy. Circulation. 2006; 114(3): 216-225, doi: 10.1161/CIRCULATIONAHA.105.583500, indexed in Pubmed: 16831987. 
5. Olivotto I, Montereggi A, Mazzuoli F, et al. Clinical utility and safety of exercise testing in patients with hypertrophic cardiomyopathy. G Ital Cardiol. 1999; 29(1): 11-19, indexed in Pubmed: 9987042.

6. Elliott PM, Anastasakis A, Borger MA, et al. Authors/Task Force members. 2014 ESC Guidelines on diagnosis and management of hypertrophic cardiomyopathy: the Task Force for the Diagnosis and Management of Hypertrophic Cardiomyopathy of the European Society of Cardiology (ESC). Eur Heart J. 2014; 35(39): 2733-2779, doi: 10.1093/eurheartj/ehu284, indexed in Pubmed: 25173338.

7. Kappenberger L, Linde C, Daubert C, et al. Pacing in hypertrophic obstructive cardiomyopathy. A randomized crossover study. PIC Study Group. Eur Heart J. 1997; 18(8): 1249-1256, indexed in Pubmed: 9458416.

8. Wigle ED, Chrysohou A, Bigelow WG. Results of ventriculomyotomy in muscular subaortic stenosis. Am J Cardiol. 1963; 11: 572-586, indexed in Pubmed: 14000611.

9. Sigwart U. Non-surgical myocardial reduction for hypertrophic obstructive cardiomyopathy. Lancet. 1995; 346(8969): 211-214, indexed in Pubmed: 7616800.

10. Kornacewicz-Jach Z, Gil R, Wojtarowicz A, et al. Alkoholowa ablacja tętnicy septalnej w kardiomiopatii przerostowej z zawężaniem drogi odplywu. Wyniki wczesne. Kardiol Pol. 1998; 48: 105-112.

11. Elliott P, Gimeno J, Tomé M, et al. Left ventricular outflow tract obstruction and sudden death in hypertrophic cardiomyopathy. Eur Heart J. 2006; 27(24): 3073; author reply 3073-4, doi: 10.1093/eurheartj/ehl383, indexed in Pubmed: 17121751.

12. Maron BJ, Olivotto I, Spirito P, et al. Epidemiology of hypertrophic cardiomyopathy-related death: revisited in a large nonreferral-based patient population. Circulation. 2000; 102(8): 858-864, indexed in Pubmed: 10952953.

13. O'Mahony C, Tome-Esteban M, Lambiase PD, et al. A validation study of the 2003 American College of Cardiology/European Society of Cardiology and 2011 American College of Cardiology Foundation/American Heart Association risk stratification and treatment algorithms for sudden cardiac death in patients with hypertrophic cardiomyopathy. Heart. 2013; 99(8): 534-541, doi: 10.1136/heartjnl-2012-303271, indexed in Pubmed: 23339826.

14. Olivotto I, Cecchi F, Poggesi C, et al. Patterns of disease progression in hypertrophic cardiomyopathy: an individualized approach to clinical staging. Circ Heart Fail. 2012; 5(4): 535-546, doi: 10.1161/CIRCHEARTFAILURE.112.967026, indexed in Pubmed: 22811549.
15. Jensen MK, Prinz C, Horstkotte D, et al. Alcohol septal ablation in patients with hypertrophic obstructive cardiomyopathy: low incidence of sudden cardiac death and reduced risk profile. Heart. 2013; 99(14): 1012-1017, doi: 10.1136/heartjnl-2012-303339, indexed in Pubmed: 23644300.

16. Ommen SR, Maron BJ, Olivotto I. Long-term effects of surgical septal myectomy on survival in patients with obstructive hypertrophic cardiomyopathy. J Am Coll Cardiol. 2005; 46: 470-476.

17. Faber L, Seggewiss H, Gleichmann U. Percutaneous transluminal septal myocardial ablation in hypertrophic obstructive cardiomyopathy: results with respect to intraprocedural myocardial contrast echocardiography. Circulation. 1998; 98(22): 2415-2421, indexed in Pubmed: 9832486.

18. Agarwal S, Tuzcu EM, Desai MY, et al. Updated meta-analysis of septal alcohol ablation versus myectomy for hypertrophic cardiomyopathy. J Am Coll Cardiol. 2010; 55(8): 823-834, doi: 10.1016/j.jacc.2009.09.047, indexed in Pubmed: 20170823.

19. Alam M, Dokainish H, Lakkis NM. Hypertrophic obstructive cardiomyopathy-alcohol septal ablation vs. myectomy: a metaanalysis. Eur Heart J. 2009; 30(9): 1080-1087, doi: 10.1093/eurheartj/ehp016, indexed in Pubmed: 19233857.

20. Liebregts M, Vriesendorp PA, Mahmoodi BK, et al. A Systematic Review and Meta-Analysis of Long-Term Outcomes After Septal Reduction Therapy in Patients With Hypertrophic Cardiomyopathy. JACC Heart Fail. 2015; 3(11): 896-905, doi: 10.1016/j. jchf.2015.06.011, indexed in Pubmed: 26454847.

21. Fernandes VL, Nielsen C, Nagueh SF, et al. Follow-up of alcohol septal ablation for symptomatic hypertrophic obstructive cardiomyopathy the Baylor and Medical University of South Carolina experience 1996 to 2007. JACC Cardiovasc Interv. 2008; 1(5): 561-570, doi: 10.1016/j.jcin.2008.07.005, indexed in Pubmed: 19463359.

22. Kuhn H, Lawrenz T, Lieder F, et al. Survival after transcoronary ablation of septal hypertrophy in hypertrophic obstructive cardiomyopathy (TASH): a 10 year experience. Clin Res Cardiol. 2008; 97(4): 234-243, doi: 10.1007/s00392-007-0616-7, indexed in Pubmed: 18071624.

23. Welge D, Seggewiss H, Fassbender D, et al. Long-term follow-up after percutaneous septal ablation in hypertrophic obstructive cardiomyopathy. Deutsche Medizinische Wochenschrift. 2008; 133: 1949-1954.

24. Maron B, Spirito P, Shen WK, et al. Implantable cardioverter-defibrillators and prevention of sudden cardiac death in hypertrophic cardiomyopathy. JAMA. 2007; 298(4): 405-412, doi: 10.1001/ jama.298.4.405. 